\title{
THEOREMS ON DIRECT SUMS OF MODULES
}

\author{
CHIN-SHUI HSÜ
}

(TO UNIVERSITY OF HONG KONG FOR THE GOLDEN JUBILEE)

The purpose of this note is to extend two test problems of $\mathrm{I}$. Kaplansky [1, Problems II and III] to the categories of modules over Dedekind rings. Problem III has been solved and extended to modules over principal ideal domains by P. M. Cohn [2] and E. A. Walker [3]. A part of our treatment is a little similar to theirs.

In what follows $\Lambda$ will stand for a Dedekind ring and modules will mean $\Lambda$ modules. For submodules $\cap$ and $U$ will be used in the lattice theoretic sense.

LEmma 1. For modules $B \subset C \subset B \oplus A$, we have $C=B \oplus(C \cap A)$.

LEMma 2. In a module $A_{1} \oplus A_{2}=B_{1} \oplus B_{2}$ if $A_{2} \cong B_{2} \cong \Lambda$, then $A_{1} \cong B_{1}$.

Proof. By Lemma $1 A_{1} \cup B_{1}=B_{1} \oplus\left(\left(A_{1} \cup B_{1}\right) \cap B_{2}\right)$. Then $A_{1} / A_{1}$ $\cap B_{1} \cong A_{1} \cup B_{1} / B_{1} \cong\left(A_{1} \cup B_{1}\right) \cap B_{2} \subset B_{2}$ is projective. Therefore $A_{1}$ $=\left(A_{1} \cap B_{1}\right) \oplus A_{1}^{\prime}$. In like manner $B_{1}=\left(A_{1} \cap B_{1}\right) \oplus B_{1}^{\prime}$. From $\left(A_{1} \cap B_{1}\right) \oplus A_{1}^{\prime} \oplus A_{2}=\left(A_{1} \cap B_{1}\right) \oplus B_{1}^{\prime} \oplus B_{2}$ we get $A_{1}^{\prime} \oplus A_{2} \cong B_{1}^{\prime} \oplus B_{2}$. As $A_{1}^{\prime} \cong\left(A_{1} \cup B_{1}\right) \cap B_{2}, B_{1}^{\prime} \cong\left(A_{1} \cup B_{1}\right) \cap A_{2}$ are isomorphic with $\Lambda$ ideals, $A_{1}^{\prime} \cong B_{1}^{\prime}$ according to $\left[4\right.$, Theorem 2]. Hence $A_{1} \cong B_{1}$.

COROLlaRY. If $A \oplus C \cong B \oplus C$ where $C$ is a finitely generated free module, the $A \cong B$.

Leммa 3. If $A \oplus C \cong B \oplus C$ where $C$ is a finitely generated projective module, then $A \cong B$.

Proof. Because we can complete $C$ to a finitely generated free module by adding a suitable module.

LEmma 4. If $P$ is a prime ideal and $A \oplus \Lambda / P^{m} \cong B \oplus \Lambda / P^{m}$, then $A \cong B$.

Proof. We may assume $A \oplus C_{1}=B \oplus C_{2}$ where $C_{1} \cong C_{2} \cong \Lambda / P^{m}$. Then

(i) $B \cap C_{1}=0 \Rightarrow B \oplus\left(\left(B \cup C_{1}\right) \cap C_{2}\right)=B \oplus C_{1} \Rightarrow\left(B \cup C_{1}\right) \cap C_{2} \cong C_{1} \Rightarrow$ $\left(B \cup C_{1}\right) \cap C_{2}=C_{2} \Rightarrow A \oplus C_{1}=B \oplus C_{1} \Rightarrow A \cong B$.

(ii) $B \cap C_{1} \neq 0$. By Lemma $1, B / B \cap C_{1} \cong\left(B \cup C_{1}\right) \cap A, C_{1} / B \cap C_{1}$ $\cong\left(B \cup C_{1}\right) \cap C_{2}$. Hence $A \oplus C_{1}=B \oplus C_{2}$ gives $A \oplus\left(C_{1} / B \cap C_{1}\right)$ $\cong\left(B / B \cap C_{1}\right) \oplus C_{2} \cong\left(\left(B \cup C_{1}\right) \cap A\right) \oplus C_{1}=B \cup C_{1}=B \oplus\left(\left(B \cup C_{1}\right) \cap C_{2}\right)$ $\cong B \oplus\left(C_{1} / B \cap C_{1}\right)$.

Received by the editors June 26, 1961. 
Then the lemma may be completed by induction on $m$.

Since every finitely generated module ever a Dedekind ring is the direct sum of a finitely generated projective module and a finite number of modules of the form $\Lambda / P^{m}$, by Lemmas 3 and 4 we have

TheOREM 1. If $A \oplus C \cong B \oplus C$ where $C$ is a finitely generated module, then $A \cong B$.

COROLlARY. The theorem above also holds for modules over commutative Noetherian hereditary rings.

This is obvious since such a ring is the direct sum of a finite number of Dedekind rings $[5,4.13]$.

LEMMA 5. If $A$ and $B$ are finitely generated torsion modules and $A \oplus A$ $\cong B \oplus B$ then $A \cong B$.

Proof. We need only to prove the case of primary modules. Then obviously $A$ and $B$ should have the same order ideal, say $P^{m}$. And then $A=A_{1} \oplus A_{2}, B=B_{1} \oplus B_{2}$ where $A_{2} \cong B_{2} \cong \Lambda / P^{m}$. By Lemma 4 $A_{1} \oplus A_{1} \cong B_{1} \oplus B_{1}$. Hence the lemma may be completed by induction.

LEMMA 6. If the ideal class group of $\Lambda$ contains no element of order 2 , then for finitely generated torsion-free modules $A \oplus A \cong B \oplus B$ implies $A \cong B$.

Proof. Obviously $A$ and $B$ have the same rank, say $r$. According to $\left[4\right.$, Theorem 1] $A \cong I_{1} \oplus \cdots \oplus I_{r}, B \cong J_{1} \oplus \cdots \oplus J_{r}$ where $I_{k}, J_{k}$ are fractionary ideals of $\Lambda$. Let $I=I_{1} \cdots I_{r}, J=J_{1} \cdots J_{r}$ then $I^{2}$ and $J^{2}$ belong to the same ideal class [4, Theorem 2]. By the assumption that the ideal class group has no element of order 2, $I$ and $J$ belong to the same class. Hence $A \cong B$ [4, Theorem 2].

THEOREM 2. The following conditions on $\Lambda$ are equivalent.

(i) There is a non principal ideal $I$ such that $I \oplus I \cong \Lambda \oplus \Lambda$.

(ii) The ideal class group contains an element of order 2.

(iii) There are principal ideals $(\alpha),(\beta),(\gamma)$ such that $(\alpha)(\gamma)=(\beta)^{2}$; $(\alpha) \cup(\gamma)=\Lambda$ and $(\alpha)$ is not the square of a principal ideal.

(iv) There are non-isomorphic finitely generated modules $A$ and $B$ such that $A \oplus A \cong B \oplus B$.

Proof. (i) $\Leftrightarrow$ (ii) follows from [4, Theorem 2].

(ii) $\Rightarrow$ (iii). If $I^{2}=(\alpha)$, then from $[6, \mathrm{Ch}$. V] $I=(\alpha, \beta)$. And then $\beta^{2}=\alpha \gamma, \alpha=\lambda \alpha^{2}+\mu \alpha \beta+\nu \beta^{2}$, i.e. $1=\lambda \alpha+\mu \beta+\nu \gamma$. Squaring and replacing $\beta^{2}$ by $\alpha \gamma$ we get $\xi \alpha+\eta \gamma=1$. Hence $(\alpha) \cup(\gamma)=\Lambda$.

If $(\alpha)=\left(\alpha^{\prime}\right)^{2}$ then $I=\left(\alpha^{\prime}\right)$ according to the unique factorization property of ideals. Hence $(\alpha)$ is not a square of a principal ideal. 
(iii) $\Rightarrow$ (ii). Let $I=(\alpha, \beta)$, then $I^{2}=(\alpha)$. And $I$ is not principal since $(\alpha)$ is not the square of a principal ideal.

(i) $\Rightarrow$ (iv) is obvious.

(iv) $\Rightarrow$ (ii) follows from Lemmas 5 and 6 .

To see that the condition "the ideal class group of $\Lambda$ contains no element of order 2," for Kaplansky's Problem II to have an affirmative answer in the category of finitely generated modules over $\Lambda$, is not superfluous, we give the following example. In the Dedekind ring $Z\left[(-5)^{1 / 2}\right]$, let $I=\left(3,2+(-5)^{1 / 2}\right)$ then $I^{2}=\left(2+(-5)^{1 / 2}\right)$ and $I$ is not principal. But the condition is still quite general, it is valid, for example, in all unique factorization Dedekind domains.

\section{REFERENCES}

1. I. Kaplansky, Infinite Abelian groups, Univ. of Michigan Press, Ann Arbor, Mich., 1954.

2. P. M. Cohn, The complement of a finitely generated direct summand of an Abelian group, Proc. Amer. Math. Soc. 7 (1956), 520-521.

3. E. A. Walker, Cancellation in direct sums of groups, Proc. Amer. Math. Soc. 7 (1956), 898-902.

4. I. Kaplansky, Modules over Dedekind rings and valuation rings, Trans. Amer. Math. Soc. 72 (1952), 327-340.

5. M. Auslander and D. A. Buchsbaum, Homological dimension in local rings, Trans. Amer. Math. Soc. 85 (1957), 390-405.

6. O. Zariski and P. Samuel, Commutative algebra, Van Nostrand, New York, 1958.

University of Hong Kong, Hong Kong 